07

\title{
Релаксация механических напряжений в эпитаксиальных пленках кубического карбида кремния на кремниевых подложках с буферным пористым слоем
}

\author{
(C) А.С. Гусев, Н.И. Каргин, С.М. Рындя, Г.К. Сафраралиев, Н.В. Сигловая, М.О. Смирнова, И.О. Соломатин, \\ А.О. Султанов, А.А. Тимофеев
}

Национальный исследовательский ядерный университет „МИФИ“, 115409 Москва, Россия

e-mail: karabi86@mail.ru

Поступило в Редакцию 30 декабря 2020 г.

В окончательной редакции 19 января 2021 г.

Принято к публикации 21 января 2021 г.

Результаты работы количественно и качественно освещают процессы релаксации напряжений несоответствия, возникающих при эпитаксии кубического карбида кремния на кремнии. Проведен анализ распределений механических напряжений в гетероструктурах $3 C-\mathrm{SiC} / \mathrm{Si}$ и $3 C-\mathrm{SiC} /$ por $-\mathrm{Si}$. Показана существенная роль пористого буферного слоя в уменьшении величины напряжений несоответствия. Данные теоретического исследования подтверждены экспериментальными значениями остаточных напряжений в образцах $3 C-\mathrm{SiC} / \mathrm{Si}$ и $3 C-\mathrm{SiC} /$ por $-\mathrm{Si}$.

Ключевые слова: карбид кремния, пористый кремний, остаточные напряжения, дислокации несоответствия.

DOI: 10.21883/JTF.2021.06.50870.353-20

\section{Введение}

Наиболее важной особенностью $3 C-\mathrm{SiC}$ является возможность его синтеза на подложках $\mathrm{Si}$ большого диаметра, что позволяет рассматривать $\mathrm{SiC}$ как привлекательный материал для приборов силовой электроники и фотоники. Однако синтез пленок $3 C-\mathrm{SiC}$ приборного качества на монокристаллическом кремнии ограничен фактором рассогласования по параметрам решеток (19.7\%) и коэффициентам теплового расширения $(8 \%)$ [1]. Напряжения несоответствия релаксируют до остаточных значений путем возникновения и перемещения дислокаций. Вследствие этого нарушается структура пленок $3 \mathrm{C}-\mathrm{SiC}$, что влияет на их электрофизические свойства. В ряде работ [2-5] авторами получены значения остаточных напряжений монокристаллических пленок $3 C-\mathrm{SiC}$, синтезированных методом эпитаксии из газовой фазы (CVD) на подложках $\mathrm{Si}$. Значения измеренных остаточных напряжений лежат в достаточно широком диапазоне от 215 до $870 \mathrm{MPa} \mathrm{и} \mathrm{не} \mathrm{могут} \mathrm{дать}$ однозначной картины процессов упругой деформации гетероструктур $3 C-\mathrm{SiC} / \mathrm{Si}$.

$\mathrm{C}$ целью устранения дефектов несоответствия и улучшения кристаллического совершенства растущего слоя при гетероэпитаксиальном выращивании $3 C-\mathrm{SiC}$ необходимо использовать буферные слои.

Эпитаксия карбида кремния на подложках $\mathrm{Si}$ с приповерхностным пористым слоем (por-Si) позволяет существенно снизить внутренние напряжения несоответствия в синтезируемых пленках [6-8]. Тем не менее в настоящее время в специальной научной литературе отсутствуют научные исследования процессов релаксации внутренних напряжений гетероэпитаксиальных пленок $3 C-\mathrm{SiC}$ буферным слоем пористого кремния. В настоящей работе приведены результаты исследований распределений механических напряжений в гетероструктурах $3 C-\mathrm{SiC} / \mathrm{Si}$ и $3 C-\mathrm{SiC} /$ por $-\mathrm{Si}$.

\section{1. Некоторые особенности упругих свойств кристаллической структуры $3 C-\mathrm{SiC}$}

Закон Гука с переходом от тензорной индексации на матричную для монокристаллов кубического политипа имеет вид:

$$
\left(\begin{array}{l}
\sigma_{11} \\
\sigma_{22} \\
\sigma_{33} \\
\sigma_{32} \\
\sigma_{31} \\
\sigma_{21}
\end{array}\right)=\left(\begin{array}{cccccc}
c_{11} & c_{12} & c_{12} & 0 & 0 & 0 \\
c_{12} & c_{11} & c_{12} & 0 & 0 & 0 \\
c_{12} & c_{12} & c_{11} & 0 & 0 & 0 \\
0 & 0 & 0 & c_{44} & 0 & 0 \\
0 & 0 & 0 & 0 & c_{44} & 0 \\
0 & 0 & 0 & 0 & 0 & c_{44}
\end{array}\right)\left(\begin{array}{c}
\varepsilon_{11} \\
\varepsilon_{22} \\
\varepsilon_{33} \\
2 \varepsilon_{32} \\
2 \varepsilon_{31} \\
2 \varepsilon_{21}
\end{array}\right)
$$

где $c_{i j}$ - константы жесткости кристалла. Величины $c_{i j}$ образуют тензор четвертого ранга. Тензор, составленный из коэффициентов $c_{i j}$, называют тензором упругой жесткости или просто тензором упругости. Различают нормальные $(i=j)$ и сдвиговые $(i \neq j)$ компоненты тензора напряжений в кубической решетке. Первый индекс компонент напряжений указывает направление компоненты в системе координат, второй - направление нормали площадки в этой же системе координат. Из принципа Неймана следует, что число независимых компонентов упругости можно свести к трем для случая 


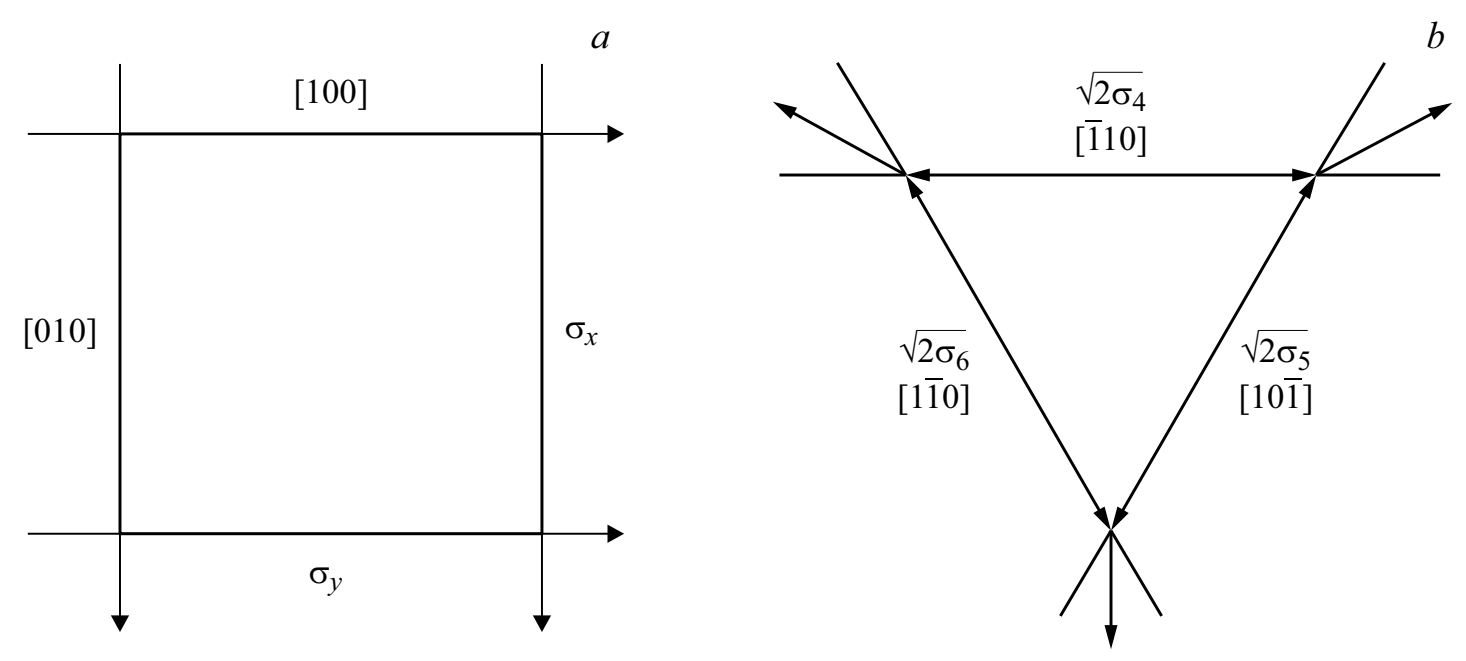

Рис. 1. Схема, иллюстрирующая приложение сил растяжения кубической решетки: $a-$ в плоскости $(001) ; b-$ в плоскости (111).

твердого тела с кубическим типом решетки. Симметрия кубической решетки обусловливает равенство коэффициентов вдоль пространственных осей и диагоналей: $c_{11}=c_{22}=c_{33} ; c_{44}=c_{55}=c_{66} ; c_{12}=c_{23}=c_{13}$. Коэффициенты, соответствующие вращательным компонентам сил, не деформирующим кубическую решетку, равны нулю.

При деформации растяжения вдоль направлений [100] и [010] (рис. 1,a) для плоскостей $\{001\}$ выражение (1) можно представить как систему линейных уравнений:

$$
\begin{aligned}
& \sigma_{11}=\sigma_{x}=c_{11} \varepsilon_{11}+c_{12} \varepsilon_{22}+c_{12} \varepsilon_{33}, \\
& \sigma_{22}=\sigma_{y}=c_{12} \varepsilon_{11}+c_{11} \varepsilon_{22}+c_{12} \varepsilon_{33}, \\
& \sigma_{33}=\sigma_{z}=c_{12} \varepsilon_{11}+c_{12} \varepsilon_{22}+c_{11} \varepsilon_{33} .
\end{aligned}
$$

Поскольку $\varepsilon_{11}=\varepsilon_{22}=\varepsilon$, а воздействие по оси $z$ отсутствует $\left(\sigma_{z}=0\right)$, то для растянутого слоя $3 C-\mathrm{SiC}$ получаем

$$
\begin{gathered}
\sigma_{x}=\sigma_{y}=M_{100} \varepsilon, \\
\varepsilon_{33}=\varepsilon_{z}=-\frac{2 c_{12}}{C_{11}} \varepsilon, \\
M_{[100]}=c_{11}+c_{12}-2 c_{12}^{2} / c_{11},
\end{gathered}
$$

где $\sigma_{x}$ и $\sigma_{y}-$ напряжения, соответствующие в системе координат, осям $x$ и $y$ соответственно, $\varepsilon=0.24541-$ деформация вдоль осей координат $x$ и $y, \varepsilon_{z}-$ деформация по оси $z, M[100]$ - биаксиальный модуль упругости, $[\mathrm{GPa}]$. Карбиду кремния кубического политипа соответствуют константы жесткости $c_{11}=290 \mathrm{GPa}$, $c_{12}=235 \mathrm{GPa}$ и $c_{44}=55 \mathrm{GPa}[9-11]$.

В случае деформации вдоль направлений [1ํㅣ, [110] и $[10 \overline{1}]$ для плоскостей $\{111\}$ закон Гука (1) сводиться к системе уравнений

$$
\begin{aligned}
& \sigma_{11}=c_{11} \varepsilon_{11}+c_{12} \varepsilon_{22}+c_{12} \varepsilon_{33}, \\
& \sigma_{22}=c_{12} \varepsilon_{11}+c_{11} \varepsilon_{22}+c_{12} \varepsilon_{33},
\end{aligned}
$$

$$
\begin{gathered}
\sigma_{33}=c_{12} \varepsilon_{11}+c_{12} \varepsilon_{22}+c_{11} \varepsilon_{33}, \\
\sigma_{32}=2 c_{44} \varepsilon_{32}, \\
\sigma_{31}=2 c_{44} \varepsilon_{31}, \\
\sigma_{21}=2 c_{44} \varepsilon_{21} .
\end{gathered}
$$

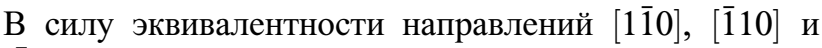
$[10 \overline{1}]$ для кубической решетки (рис. $1, b)$, связь напряжения растяжения и деформации решетки можно привести к виду:

$$
\begin{gathered}
\sigma_{[\overline{1} 10]}=M_{111} \varepsilon_{32}, \\
M_{[\overline{1} 10]}=2 \sqrt{2} c_{44},
\end{gathered}
$$

где $\varepsilon_{32}=0.24554$.

Также на этапе охлаждения гетероструктуры $3 \mathrm{C}-\mathrm{SiC} / \mathrm{Si}$ до комнатной температуры пленка карбида кремния „стягивает“ подложку кремния, что приводит к деформации изгиба. Согласно теории упругости, деформация изгиба характеризуется радиусом кривизны $R$. Наличие в гетероструктуре $3 C-\mathrm{SiC} / \mathrm{Si}$ дислокаций, пор и других дефектов структуры релаксирует напряжения несоответствия, что приводит к увеличению значения радиуса кривизны.

\section{2. Расчет значений остаточных напряжений по спектрам комбинационного рассеяния}

Методом эпитаксии из газовой фазы (CVD) был получен экспериментальный ряд гетероструктур $3 C-\mathrm{SiC} / \mathrm{Si}$ и $3 C-\mathrm{SiC} /$ por $-\mathrm{Si}$. Синтез пленок $3 C-\mathrm{SiC}$ проводил-

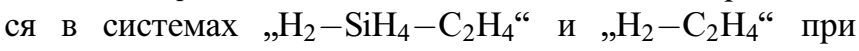
температуре $1100-1300^{\circ} \mathrm{C}$. Использовались подложки монокристаллического кремния с ориентацией поверхности (111) и (100), а также образцы, содержащие 

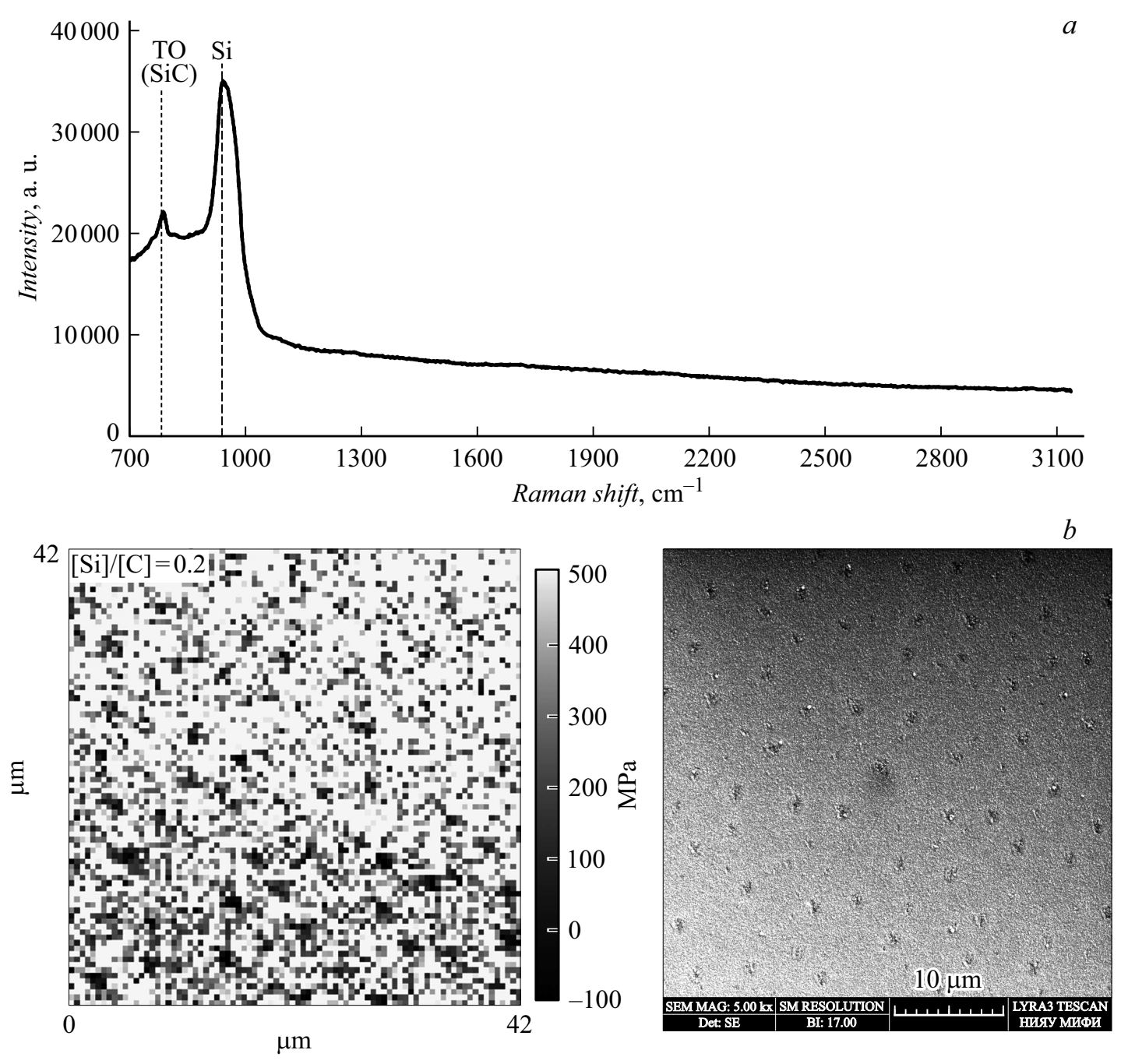

Рис. 2. $a-$ пример рамановского спектра экспериментальных гетероструктур $3 C-\mathrm{SiC} / \mathrm{Si} ; b-$ сравнение карт распределения остаточных напряжений и РЭМ изображений поверхности экспериментальных образцов $3 C-\mathrm{SiC} / \mathrm{Si}$. Ориентация поверхности подложек - (111). Размер одного пикселя $-0.5 \mu \mathrm{m}$.

слой мезопористого кремния. Мезопористые слои получены методом анодирования на подложках кремния с ориентацией поверхности (100). Величина остаточных напряжений в экспериментальных образцах оценивалась по смещению в длинноволновую область пиков $3 C-\mathrm{SiC}$ в спектрах комбинационного (рамановского) рассеяния. Смещение оптического (ТО) пика пленки карбида кремния $\omega_{\text {то }}$ от значения $\omega=796.5 \pm 0.3 \mathrm{~cm}^{-1}$ объясняется деформацией несоответствия. Остаточное механическое напряжение пленок $3 C-\mathrm{SiC}$ определялось с помощью выражения [12]:

$$
\omega_{\mathrm{TO}}\left(\mathrm{cm}^{-1}\right)=(796.5 \pm 0.3)+(3730 \pm 30) \frac{\Delta a}{a_{0}},
$$

где $\Delta a / a_{0}$ - деформация кристаллической решетки $3 C-\mathrm{SiC}$.

Спектры комбинационного рассеяния получены с помощью спектрометра Confotec NR 500. В качестве возбуждающего излучения использовался лазерный ис- точник с длиной волны $532 \mathrm{~nm}$. Мощность излучения, падающего на поверхность пластины, составляет около $27 \mathrm{~mW}$. Измерения проводились путем сканирования лазерным пучком поверхности экспериментальных образцов. Сканирование по длине волн осуществлялось с помощью дифракционной решетки с плотностью штрихов $1800 \mathrm{~mm}^{-1}$. Рассеянное излучение регистрировалось ПЗС-матрицей. Время экспозиции в каждой точке составляет $4 \mathrm{~s}$.

Для проведения исследований были получены спектральные карты с размерами $30 \times 30 \mu \mathrm{m}$. Карта представляет собой набор спектров, полученных при поточечном прохождении лазерного пучка по поверхности пластины. Шаг между точками составлял $0.5 \mu \mathrm{m}$, размер лазерного пятна $0.6 \mu \mathrm{m}$.

Спектральные карты были переработаны в программной среде Python в карты распределения остаточных напряжений. На рис. 2 представлены примеры спектральной линии и сопоставления карты остаточных напряже- 
Значения остаточных напряжений экспериментальных образцов $3 C-\mathrm{SiC} / \mathrm{Si}$

\begin{tabular}{|c|c|c|}
\hline \multirow{2}{*}{$\begin{array}{c}\text { Тип } \\
\text { подложки }\end{array}$} & \multicolumn{2}{|c|}{ Значения остаточных напряжений, МРа } \\
\hline & $\begin{array}{c}\text { Определенные по смещению ТО линий } \\
\text { спектров комбинационного рассеяния }( \pm 0.5 \mathrm{MPa})\end{array}$ & $\begin{array}{c}\text { Определенные по спектрам рентгеновской } \\
\text { дифрактометрии }( \pm 0.5 \mathrm{MPa})\end{array}$ \\
\hline Si $(100)$ & $\begin{array}{l}925.8 \\
811.0\end{array}$ & - \\
\hline $\begin{array}{c}\mathrm{Si}(100) \\
\text { с пористым } \\
\text { слоем }\end{array}$ & $\begin{array}{l}466.7 \\
504.9 \\
543.2\end{array}$ & $\begin{array}{c}449.3 \\
571.1 \\
-\end{array}$ \\
\hline $\mathrm{Si}(111)$ & 752.5 & - \\
\hline
\end{tabular}
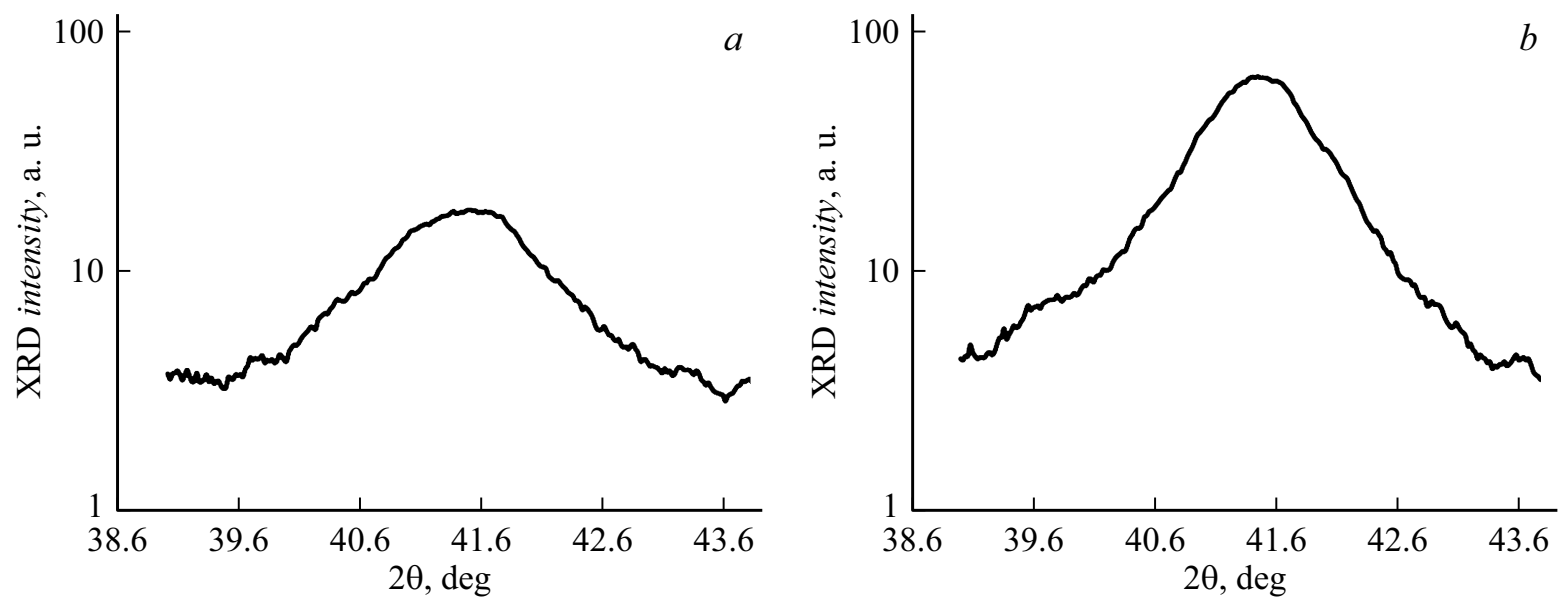

Рис. 3. Рентгенодифракционные пики экспериментальных образцов $3 C-\mathrm{SiC} / \mathrm{Si}$, синтезированных на подложках с ориентацией поверхности (111).

ний с РЭМ изображением экспериментальных образцов, выращенных на подложках кремния с ориентацией поверхности (111). На спектральной линии (рис. 2, a) наблюдается пик $3 C-\mathrm{SiC}$ на частоте $797 \mathrm{~cm}^{-1}$, соответствующий поперечным оптическим (ТО) фононам. Визуально распределение остаточных напряжений на поверхности образца представляет собой темные изолированные зоны, соответствующие сжатию материала (отрицательные значения, [MPa]) в окружении областей, где преобладает растяжение (положительные значения, $[\mathrm{MPa}])$. Полученная картина распределения остаточных напряжений имеет сходство с распределением пор в плоскости сопряжения эпитаксиальной пленки и подложки, т.е. зоны, где преобладает сжатие, относятся к участкам слоя $\mathrm{SiC}$ над порами. Данные расшифровки карт приведены в таблице. Значения остаточных напряжений $\sigma_{r e s}$ в определенных пределах погрешности соответствуют значениям, взятым из литературных источников [2-5].

Также значения остаточных напряжений синтезированных пленок находили по спектрам рентгеновской дифрактометрии. Расчет остаточных напряжений основан на представлении об изменении межплоскостных расстояний в кристалле при упругой деформации. При наличии упругих напряжений в пленке карбида кремния система плоскостей $(h k l)$ изменяет строго определенное значение межплоскостного расстояния $d_{0}$. Величина остаточных напряжений определяется по соответствующему значению относительной деформации $\varepsilon$. Значение остаточных напряжений в пленке определяется следующей формулой:

$$
\sigma_{r e s}=\frac{d_{0}-d}{d_{0}} \frac{M_{h k l}}{\mu}
$$

где $d_{0},[\mathrm{~nm}]-$ межплоскостное расстояние в монокристалле $3 C-\mathrm{SiC} ; d,[\mathrm{~nm}]$ - межплоскостное расстояние в упруго-напряженной пленке $3 C-\mathrm{SiC} ; M_{h k l}-$ модуль упругости для плоскостей $\{100\}$ и $\{111\} 3 C-\mathrm{SiC} ; \mu-$ коэффициент Пуассона $[9,13]$. Межплоскостные расстояния определялись анализом рентгенодифракционных спектров экспериментальных образцов, с помощью условия Вульфа-Брэгга.

Измерение рентгенодифракционных спектров экспериментальных образцов проводилось на дифрактометре „Ultima IV“ (Rigaku) с использованием $\mathrm{Cu} K_{\alpha}$-излучения $(\lambda=0.15406 \mathrm{~nm})$ по двухкристальной схеме. На рис. 3 приведены примеры снятых спектральных линий, первого порядка от плоскостей отражения $\{100\} 3 C-\mathrm{SiC}$. 


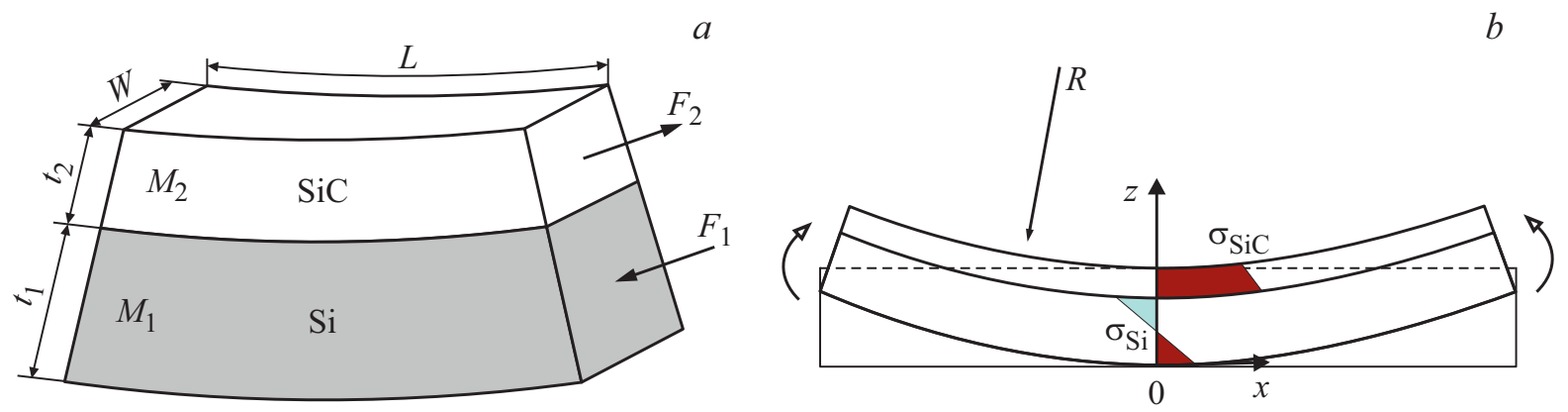

Рис. 4. Схема изогнутой двухслойной структуры $3 C-\mathrm{SiC} / \mathrm{Si}$.

Расчет остаточных напряжений по формулам (3) и (4) дает значения для структур $3 C-\mathrm{SiC} / \mathrm{Si}$ и $3 C-\mathrm{SiC} /$ por $-\mathrm{Si}$, приведенные в таблице.

При этом степени релаксации пленки $R_{\text {rel }}>0.95$. Степень релаксации определяется выражением

$$
R_{r e l}=1-\frac{\varepsilon_{r e s}}{\varepsilon_{\max }}
$$

где $\varepsilon_{\text {res }}=\sigma_{\text {res }} / E_{h k l}, \varepsilon_{\max }=\varepsilon_{0}$.

\section{3. Оценка значения напряжений несоответствия}

С учетом сказанного выше, распределение механического напряжения в структуре $3 \mathrm{C}-\mathrm{SiC} / \mathrm{Si}$ можно построить с использованием модели, предложенной в [14,15]. В рамках данной модели рассматривается деформация двухслойной структуры, шириной $W$, постоянными упругости $M_{i}$ и толщинами слоев $t_{i}$, радиусом кривизны $R$, как показано на рис. 4, $a$. На систему действуют силы растяжения-сжатия $F_{i}$. Здесь $i=1$ и 2 обозначают подложку кремния и слой карбида кремния соответственно.

Система координат выбрана таким образом, что оси $x$ и $y$ параллельны поверхности подложки, ось $z$ ориентирована по нормали к поверхности гетероструктуры, как показано на рис. 4, $b$. Таким образом, напряжение по оси $z$ принято равным нулю. Для сведения задачи к одному измерению принимается, что длина выделенного элемента $L \gg W$, в этом случае изгибом по ширине можно пренебречь.

Построение эпюр напряжений слоев происходит с помощью условий равновесия сил $F_{i}$. Условия равновесия представлены выражениями:

$$
F_{1}-F_{2}=0
$$

Согласно уравнениям (2), напряжения растяжениясжатия определяются выражениями

$$
\sigma_{p-c}=\frac{F_{s-c}}{W t_{i}}=M_{i} \varepsilon_{i},
$$

где $F_{s-c}-$ силы растяжения-сжатия.
При сравнении сил $F_{s-c}$, создающих деформации равной величины, для подложек кремния соотношение $F_{(s-c) 1} / F_{(s-c) 2} \sim 10^{3}$. В связи с этим в рамках рассматриваемой модели деформацией сжатия подложки можно пренебречь.

Деформацию слоя $3 C-\mathrm{SiC}(i=2)$ можно представить суммой деформаций решетки $\varepsilon_{0}=\Delta a / a_{0}$, несоответствия коэффициентов теплового расширения $\Delta a$, изгиба гетероструктуры, а также составляющей, определяемой образованием дислокаций. Согласно теории упругости [11], деформация, обусловленная изгибом, задается как $\varepsilon_{\text {bend }}=t_{i} / 2 R$. Поскольку сжимаемость подложки не учитывается, то ее деформация определяется изгибом. Таким образом, деформации на гетерогранице можно представить в виде

$$
\varepsilon_{1}=\frac{t_{1}}{2 R}, \quad \varepsilon_{2}=\varepsilon_{0}+\Delta \alpha \Delta T-\rho_{0} b-\varepsilon_{p h}-\frac{t_{2}}{2 R},
$$

где $\varepsilon_{0}$ - деформация пленки $\mathrm{SiC}[6] ; \Delta \alpha-$ разность коэффициентов теплового расширения слоев материалов 1 и $2, \Delta T=1280^{\circ} \mathrm{C} ; \rho_{0}=10^{6},\left[\mathrm{~cm}^{-1}\right]$ и $b-$ линейная плотность и модуль вектора Бюргерса системы дислокаций, введенных в пленку. Линейная плотность дислокаций представляет собой число дислокационных линий, пересекающих отрезок направления длиной $1 \mathrm{~cm}$. Для плоскостей (111) и (001) вектор Бюргерса равен $a_{\mathrm{SiC}} / \sqrt{2}$ и $a_{\mathrm{Sic}} / 2 \sqrt{2}$ соответственно. Слагаемое $\varepsilon_{p h}$ характеризует релаксацию напряжений на границах доменов. С учетом выражений (8) приложенные силы:

$$
\begin{gathered}
F_{1}=M_{1} W \frac{t_{1}^{2}}{2 R}, \\
F_{2}=M_{2} t_{2} W\left(\varepsilon_{0}+\Delta \alpha \Delta T-\rho_{0} b-\varepsilon_{p h}-\frac{t_{2}}{2 R}\right) .
\end{gathered}
$$

Уравнения (6) и (8) дают значение для радиуса кривизны структуры:

$$
R=\frac{M_{1} t_{1}^{2}+M_{2} t_{2}^{2}}{2 M_{2} t_{2}\left(\varepsilon_{0}+\Delta \alpha \Delta T-\rho_{0} b-\varepsilon_{p h}\right)} .
$$

Аналитическое выражение механических напряжений в гетероструктурах $3 \mathrm{C}-\mathrm{SiC} / \mathrm{Si}$ можно вывести из анализа деформации изгиба (рис. 5). Двумя сечениями 


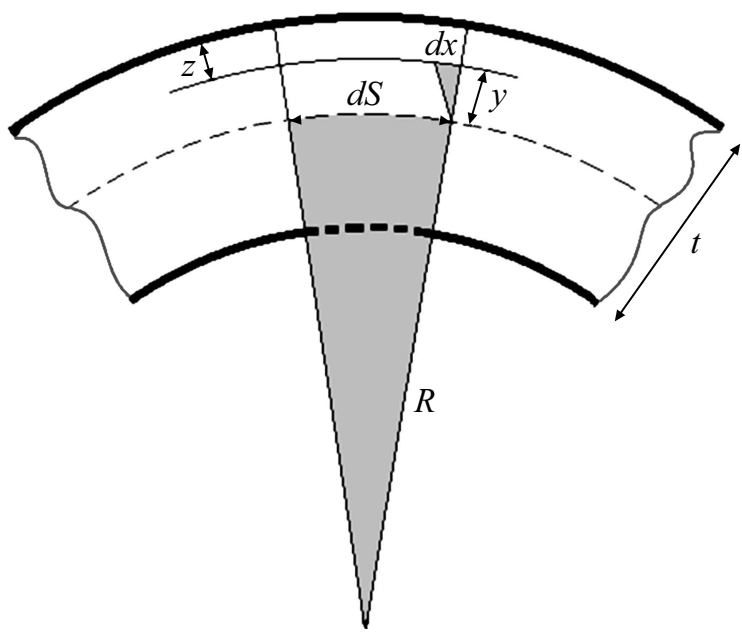

Рис. 5. Схема участка пластины, подверженной деформации изгиба.

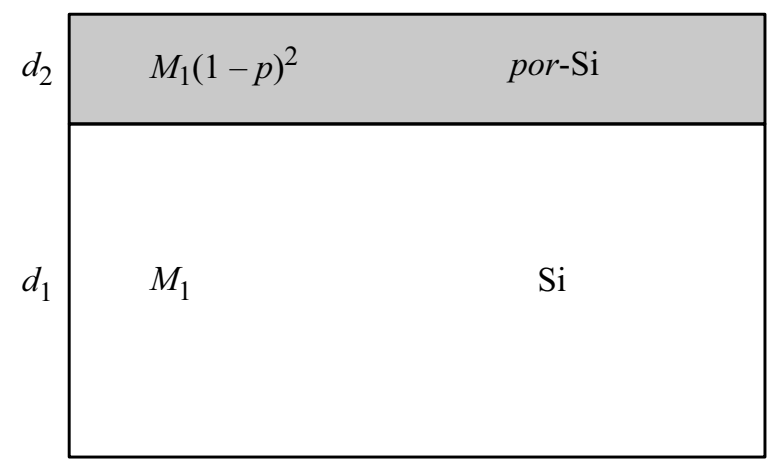

Рис. 6. Значения модуля упругости в подложке $\mathrm{Si}$ с пористым слоем.

выделим малый элемент пластины. Нейтральная линия (пунктирная) делит сечение на две части, в одной из которых действуют растягивающие внутренние силы, а в другой - сжимающие.

Длина отрезка нейтрального слоя $d S$. При деформации изгиба длина нейтральной линии не меняется. Линия, находящаяся на расстоянии $y$, изменит свою длину на величину $d x$. При малых значениях $d S$ имеет место следующее соотношение:

$$
\frac{d x}{d S}=\frac{y}{R} .
$$

Левая часть формулы (11) есть относительная деформация $\varepsilon$. Если присвоить деформации растяжения знак „+““ и ввести координату $z$, с нулевым значением на выпуклой границе, то выражение для деформации принимает вид

$$
\varepsilon=\frac{y}{R}=\frac{1}{R}\left(\frac{t}{2}-z\right)
$$

Напряжение в $i$-м слое $\sigma_{i}(z)$ задается суммой составляющих, вызванных приложенной силой $F_{i}$, и деформа- цией изгиба [15]. Для подложки кремния

$$
\sigma_{1}(z)=\frac{M_{1}}{R}\left(t_{1}-z\right),
$$

где $z$ - координата в $i$-м слое, измеренная со „дна“. В свою очередь, для слоя $3 C-\mathrm{SiC}$

$$
\sigma_{2}(z)=M_{2}\left(\varepsilon_{0}+\Delta \alpha \Delta T-\rho_{0} b-\varepsilon_{p h}\right)+\frac{M_{2}}{R}\left(\frac{t_{2}}{2}-z\right) .
$$

Ранее авторами $[6,8]$ экспериментально показано, что применение пористого кремния в качестве буферного слоя в гетероструктурах $3 C-\mathrm{SiC} / \mathrm{Si}$, позволяет снизить значение остаточных напряжений. Для получения выражений, описывающих остаточные напряжения в гетроструктурах $3 C-\mathrm{SiC} /$ por-Si необходимо ввести указанные ниже корректировки.

Построение эпюр напряжений при наличии буферного мезопористого слоя проводится по аналогичной схеме с помощью уравнений равновесия. Условие равновесия также описывается уравнением (6). Выражение для деформации слоя карбида кремния можно получить введением упрощенной модели пористого слоя как массива вертикальных цилиндрических пор в сплошном материале. Подобная модель представлена в работе [16]. $\mathrm{B}$ описываемой структуре площадь сопряжения пленки карбида кремния и подложки определяется пористостью буферного слоя $(p)$. Для учета влияния пористости на деформацию $\varepsilon_{i}$ введен поправочный коэффициент $(1-p)^{2}[17]$. Данный коэффициент характеризует среднюю площадь сопряжения $3 C-\mathrm{SiC} / \mathrm{Si}$. В таком случае деформации на гетерогранице

$$
\varepsilon_{1}=\frac{t_{1}}{2 R}, \quad \varepsilon_{2}=\left(\varepsilon_{0}+\Delta \alpha \Delta T-\rho_{0} b-\varepsilon_{p h}\right)(1-p)^{2}-\frac{t_{2}}{2 R} .
$$

Значение постоянной упругости подложки $M_{1}$ меняется скачкообразно на границе раздела „сплошной материал-пористый слой“. Изменение модуля упругости схематически представлено на рис. 6. Значение постоянной упругости в пористом слое равно $M_{1}(1-p)^{2}$.

В рассматриваемой модели можно наблюдать два крайних условия: $d_{2} \ll d_{1}$ и $d_{2} \gg d_{1}$. В первом случае, когда толщина пористого слоя пренебрежимо мала, можно принять общее значение постоянной упругости подложки равным $M_{1}$. Тогда решение уравнений равновесия дает следующее выражение для радиуса кривизны гетероструктуры:

$$
R=\frac{M_{1} t_{1}^{2}+M_{2} t_{2}^{2}}{2 M_{2} t_{2}\left(\varepsilon_{0}+\Delta \alpha \Delta T-\rho_{0} b-\varepsilon_{p h}\right)(1-p)^{2}} .
$$

Во втором предельном случае, при $d_{2} \gg d_{1}$, выражение для радиуса кривизны принимает вид

$$
\begin{aligned}
R= & \frac{M_{1} t_{1}^{2}}{2 M_{2} t_{2}\left(\varepsilon_{0}+\Delta \alpha \Delta T-\rho_{0} b-\varepsilon_{p h}\right)} \\
& +\frac{t_{2}}{2\left(\varepsilon_{0}+\Delta \alpha \Delta T-\rho_{0} b-\varepsilon_{p h}\right)(1-p)^{2}} .
\end{aligned}
$$



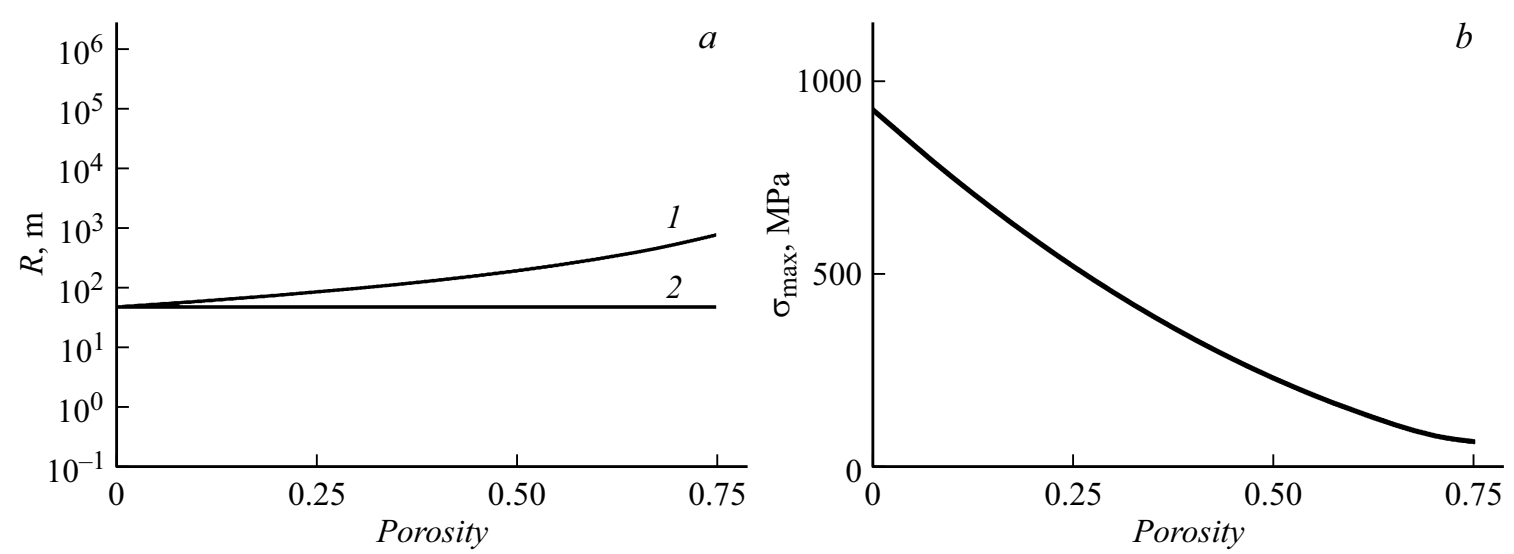

Рис. 7. $a-$ вид теоретической зависимости $R(p): 1-$ при $d_{2} \ll d_{1}, 2-$ при $d_{2} \gg d_{1} ; b-$ вид теоретической зависимости остаточного напряжения в пленке $3 \mathrm{C}-\mathrm{SiC}$.
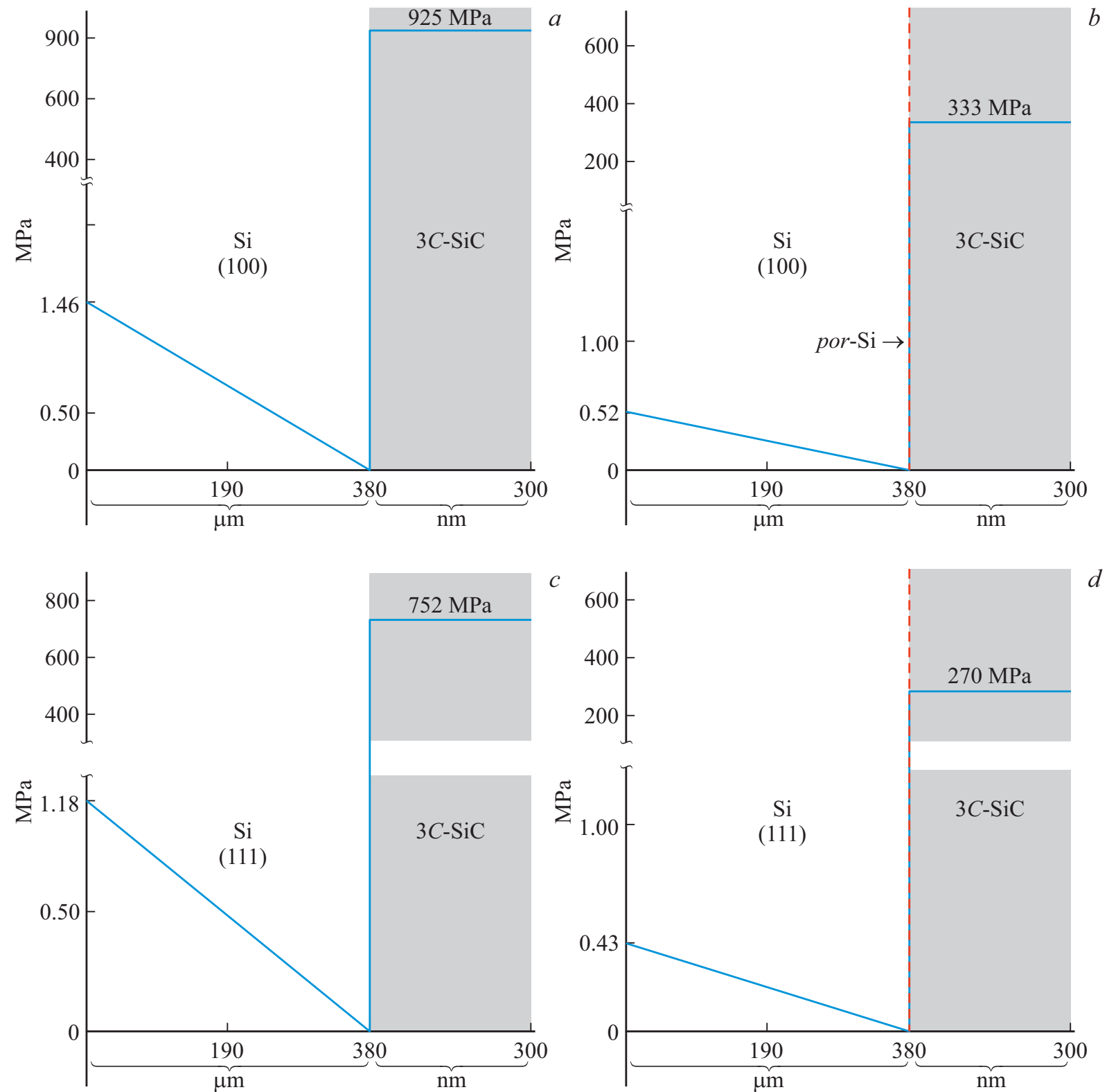

Рис. 8. Эпюры напряжений несоответствия: $a, c-$ в объеме гетероструктуры $3 C-\mathrm{SiC} / \mathrm{Si} ; b, d-$ в объеме гетероструктуры $3 C-\mathrm{SiC} / \mathrm{Si}$ с применением буферного слоя пористого кремния. Эпюры построены для подложек с ориентацией поверхности (100) и (111). 
Зависимость значения радиуса кривизны $R(p)$ и максимального остаточного напряжения $\sigma(p)$ структуры $3 C-\mathrm{SiC}$ por $-\mathrm{Si}$, и ориентация поверхности (100), приведены на рис. 7. Участки кривых $R(p)$ и $\sigma(p)$ находятся в диапазоне технологически достижимых значений пористости $(0 \leq p \leq 0.75)$. Поведение величины радиуса кривизны позволяет рассматривать условие $d_{2} \ll d_{1}$ как наиболее оптимальное для пленок $3 C-\mathrm{SiC}$ на подложках кремния с пористым слоем. В точке $p=0.4$ значение остаточного напряжения падает в $e$ раз.

С помощью выражений (10)-(14) можно построить эпюры внутренних напряжений структур $3 C-\mathrm{SiC} / \mathrm{Si}$ и $3 C-\mathrm{SiC} /$ por $-\mathrm{Si}$ для значения толщины слоя $3 C-\mathrm{SiC}$ $t_{2}=300 \mathrm{~nm}$. Вид эпюр приведен на рис. 8. Значение пористости буферного слоя $p=0.4$.

Построенные эпюры напряжения показывают что, применение буферного слоя por-Si снижает механические напряжения примерно на 64\%. Полученные значения остаточных напряжений соответствуют экспериментальным данным. Также следует заметить, что рассчитанные значения напряжений значительно меньше предела упругости монокристаллического карбида кремния $\sigma_{e l}=21 \mathrm{GPa}$ [18]. Также получены оценочные значения вклада границ зерен в уменьшение деформации пленок $\varepsilon_{p h}$. Для случая подложек кремния с ориентацией поверхности (100) и (111) $\varepsilon_{p h}$ равна $4.38 \cdot 10^{-2}$ и $4.78 \cdot 10^{-2}$ соответственно.

Таким образом, эпюры механических напряжений подтверждены расчетом остаточных напряжений в экспериментальных образцах гетероструктур $3 C-\mathrm{SiC} / \mathrm{Si}$ и $3 C-\mathrm{SiC} /$ por $-\mathrm{Si}$.

Гетероэпитаксиальный рост пленки $3 C-\mathrm{SiC}$ на подложке кремния с мезопористым буферным слоем происходит как на поверхности сопряжения со стенками остова, так и над порами. Образование $3 C-\mathrm{SiC}$ над порами идет путем их зарастания. Гетероэпитаксия на поверхности сопряжения со стенками пор, как и в случае роста пленки $3 C-\mathrm{SiC}$ на сплошном материале подложки, проходит под влиянием факторов несоответствия по параметрам кристаллических решеток и коэффициентов теплового расширения, приводящих к образованию дислокаций несоответствия.

В гетероэпитаксиальной структуре $3 C-\mathrm{SiC} / \mathrm{Si}$ линейную плотность дислокации можно представить выражением

$$
\rho=\frac{\rho_{0}}{\left(1+\frac{r^{2}}{l^{2}}\right)^{2}},
$$

где $r$ - средний диаметр пор, $l$ - толщина стенок остова, $\rho_{0},\left[\mathrm{~cm}^{-1}\right]-$ максимальная линейная плотность дислокаций.

Выражение (18) истинно при значениях толщины стенок пор $l>1 / \rho_{0}$. Согласно (18), с увеличением толщины стенок пористого слоя вклад факторов несоответствия решеток при гетероэпитаксии $3 C-\mathrm{SiC}$ растет и плотность дислокаций стремится к значению $\rho_{0}$. Для случая $l<1 / \rho_{0}$ в рамках предложенной модели ожидается рост пленки $3 C-\mathrm{SiC}$ без образования дислокаций.

\section{Заключение}

В работе представлена модель процесса релаксации напряжений несоответствия в гетероструктурах 3C-SiC/Si.

На основе предложенного подхода к описанию процессов релаксации напряжений несоответствия удалось получить аналитические выражения, отражающие влияние факторов толщины и пористости буферного слоя на распределение механических напряжений и деформаций в системе $3 C-\mathrm{SiC} /$ por-Si. Получены оценочные значения параметра $\varepsilon_{p h}$ характеризующего вклад границ зерен эпитаксиальных пленок $3 C-\mathrm{SiC}$ в релаксацию упругих напряжений. Для пленок $3 C-\mathrm{SiC}$ на подложках с ориентацией поверхности (100) и (111) $\varepsilon_{p h}$ равна $4.38 \cdot 10^{-2}$ и $4.78 \cdot 10^{-2}$ соответственно.

Применяя методику оценки величины остаточного напряжения по смещению линий на рамановских и рентгенодифракционных спектрах, получены экспериментальные значения $\sigma_{\text {res }}$, согласующиеся с результатами теоретического расчета, а также данными, взятыми из литературных источников. Расчетные данные подтверждены результатами экспериментальной оценки остаточных напряжений для образцов, синтезированных на поверхностях $\mathrm{Si}(100)$ и $\mathrm{Si}(111)$, а также $\mathrm{Si}(100)$ c мезопористым слоем. Исследования показали, что применение буферного слоя мезопористого кремния приводит к уменьшению примерно на 64\% величины остаточных напряжений на гетерогранице $3 C-\mathrm{SiC} / \mathrm{Si}$.

\section{Благодарности}

Работа проведена с применением оборудования центра коллективного пользования „Гетероструктурная СВЧ-электроника и физика широкозонных полупроводников“ Национального исследовательского ядерного университета МИФИ.

\section{Конфликт интересов}

Авторы заявляют, что у них нет конфликта интересов.

\section{Список литературы}

[1] А.А. Лебедев, Е.В. Калинина, В.В. Козловский. Поверхность. Рентгеновские, синхротронные и нейтронные исследования, (4), 77 (2018). DOI: $10.7868 / \mathrm{S} 0207352818040121$

[2] F. Liu, J. Chu, C. Carraro, R. Maboudian. J. Appl. Phys., (106), 013505 (2009). DOI: 10.1063/1.3157184

[3] A.A. Volinsky, G. Kravchenko, P. Waters, J.D. Reddy, C. Locke, C. Frewin, S.E. Saddow. Mater. Res. Society Symposia Proceed., 1069 (2009). DOI: $10.1557 /$ PROC-1069-D03-05

[4] D.N. Talwar, L. Wan, C.C. Tin, Z.C. Feng. J. Mater. Sci. Eng., 6 (2), 1 (2017). DOI: 10.4172/2169-0022.1000324 
[5] H. Mukaida, H. Okumura, J.H. Lee, H. Daimon, E. Sakuma, S. Misawa, K. Endo, S. Yoshida. J. Appl. Phys., 62, 254 (1987). DOI: 10.1063/1.339191

[6] Р.С. Телятник, А.В. Осипов, С.А. Кукушкин. ФТТ, 57 (1), 153 (2015). [R.S. Telyatnik, A.V. Osipov, S.A. Kukushkin. Phys. Solid State, 57, 162 (2015). DOI: $10.1134 / \mathrm{S} 106378341501031 \mathrm{X}]$

[7] О.М. Сресели, Д.Н. Горячев, В.Ю. Осипов, Л.В. Беляков, С.П. Вуль, И.Т. Серенков, В.И. Сахаров, А.Я. Вуль. ФТП, 36 (5), 604 (2002). [O.M. Sreseli, D.N. Goryachev, V.Yu. Osipov, L.V. Belyakov, S.P. Vul', I.T. Serenkov, V.I. Sakharov, A.Ya. Vul'. Semiconductors, 36 (5), 574 (2002). DOI: $10.1134 / 1.1478551]$

[8] N.I. Kargin, A.O. Sultanov, A.V. Bondarenko, V.P. Bondarenko, S.V. Red'ko, A S. Ionov. Russ. Microelectronics, 43 (8), 531 (2014). DOI: 10.1134/S106373971408006X

[9] A. Kelly, G.W. Groves. Crystallography and crystal defects (Longman, London, 1970)

[10] Электронный ресурс. Режим доступа: http://www.ioffe.ru/ SVA/NSM/Semicond/SiC/mechanic.html

[11] Х. Хан. Теория упругости. Основы линейной теории и ее применение, пер. с нем. (М., 1988)

[12] D. Olego, M. Cardona, P. Vogl. Phys. Rev. B, 25 (6), 3878 (1982). DOI: https://doi.org/10.1103/PhysRevB.25.3878

[13] С.С. Горелик, Л.Н. Расторгуев, Ю.А. Скаков. Рентгенографический и электроннооптический анализ (практическое руководство) (Металлургия, М., 1970), с. 126.

[14] R.H. Saul. J. Appl. Phys., 40, 3273 (1969). https://doi.org/10.1063/1.1658174

[15] G.H. Olsen, M. Ettenberg. J. Appl. Phys., 48, 2543 (1977). https://doi.org/10.1063/1.323970

[16] A.S. Gusev, N.I. Kargin, S.M. Ryndya, G.K. Safaraliev, N.V. Siglovaya, A.O. Sultanov, A.A. Timofeev. J. Surf. Investigation: X-Ray, Synchrotron and Neutron Techniq., 13 (2), 280 (2019). DOI: 10.1134/S1027451019020083

[17] И.Ю. Смолина, М.О. Еремин, П.В. Макаров, С.П. Буякова, С.Н. Кульков, Е.П. Евтушенко. Вестник Томского гос. ун-та., 5, 78 (2013).

[18] C.M. Su, A. Fekade, M. Spencer, M. Wuttig. J. Appl. Phys., 77, 1280 (1995). https://doi.org/10.1063/1.359579 